\title{
Psychometric properties of the self-rating organization scale with adult samples
}

\author{
This article was published in the following Dove Press journal: \\ Neuropsychiatric Disease and Treatment \\ 31 October 2016 \\ Number of times this article has been viewed
}

\author{
Toshinobu Takeda' \\ Yui Tsuji² \\ Mizuho Ando ${ }^{3}$ \\ 'Department of Clinical Psychology, \\ Ryukoku University, Kyoto, ${ }^{2}$ School of \\ Psychological Science, Health Sciences \\ University of Hokkaido, Sapporo, \\ ${ }^{3}$ Graduate School of Comprehensive \\ Human Sciences, University \\ of Tsukuba, Tsukuba, Japan
}

\begin{abstract}
Organization skills are defined broadly to include both materials and temporal features. Given its symptoms and neurobiological features, attention-deficit hyperactivity disorder (ADHD) should be susceptible to impairment in organization. A valid organization scale is imperative to assess and intervene individuals with ADHD. However, there is no validated organization scale in Japan. Referring to existing scales and clinical experience, the self-rating organization scale (SOS) was developed and tested in terms of its psychometric properties with 1,017 adults and students including 47 adults with ADHD. Additionally, cutoffs for disorganization were set for clinical utility. Three factors (materials disorganization, temporal disorganization, and mess) were extracted by factor analyses. The index for reliability and validity of the SOS was acceptable. The factor "mess" could reflect the unique aspect of the Japanese environment. Further study is needed to enhance the clinical utility of the SOS.
\end{abstract}

Keywords: adult, attention-deficit hyperactivity disorder, organization, scale

\section{Introduction}

Organization skills are defined broadly to include both materials (eg, school or office materials) and temporal (eg, long-term planning and scheduling) features. Across all developmental levels, these problems with organization lead to academic, occupational, and economic difficulties. ${ }^{1}$ Among a bunch of psychiatric disorders, attention-deficit hyperactivity disorder (ADHD) is one of the most susceptible conditions to difficulty in organization.

ADHD is characterized by a persistent pattern of inattention and/or hyperactivityimpulsivity that interferes with social, academic, and vocational functioning or development. ${ }^{2}$ A high prevalence of ADHD in adults (3.4\%) as well as children (7.1\%) has been reported throughout the world. ${ }^{3}$ This means that interventions for both child and adult ADHD are in high demand. An accurate assessment of symptoms or impairments is imperative to implement an appropriate therapeutic intervention.

Among the nine inattentive symptoms presented in the diagnostic criteria A for ADHD in the Diagnostic and Statistical Manual of Mental Disorders, fifth edition (DSM-5), three symptoms evidently relate to organization of objects or time: symptom e "often has difficulty organizing tasks and activities", symptom g "often loses things necessary for tasks or activities", and symptom $i$ "is often forgetful in daily activities". ${ }^{2}$ According to the $D S M-V$, these symptoms are manifested in daily life as messy; poor time management; losing school materials, pencils, books, tools, wallets, keys, paperwork, eyeglasses, and mobile phones; and keeping appointments.

Several neuropsychological models for ADHD well explain these materials disorganization (MD) and temporal disorganization (TD) in ADHD. For example, 
Barkley $^{4}$ proposed a conceptual model that links behavioral inhibition with the performance of four executive functions. Among these four executive functions (working memory, self-regulation of affect/motivation/arousal, internalization of speech, and reconstitution), working memory is most closely related to organization (eg, holding events in mind, prospective function, sense of time, and cross-temporal organization of behavior) and substantially contributes to disorganization of objects and/or time in ADHD. ${ }^{4}$

Although psychopharmacology ameliorates many of the core symptoms of ADHD and some ADHD-related impairments, there is minimal evidence to suggest that stimulant medications promote improvement in organizational skills. ${ }^{5}$ For example, in a cognitive remediation program for adults with ADHD, no significant differences in improvement were found when comparing participants taking stimulant medications and undedicated participants. ${ }^{6}$ Additionally, Baldwin et $\mathrm{al}^{6}$ did not observe any effect of methylphenidate on timeproduction performance in children with ADHD. ${ }^{7}$

Instead, there are a dozen of studies that showed the effectiveness in psychosocial intervention for organization in response to this "Pills Don't Teach Skills" situation. Landberg et $\mathrm{al}^{1}$ reviewed the then existing 18 studies on organizing interventions for either children or adults. Among these 18 studies, 12 studies reported the effect size of improvement in score of certain measures and most of them showed from middle-to-large effect sizes. To measure change in organization skills, these researchers employed, for example, classroom preparation skills, homework completion checklist, self-ratings of time management and task completion, and self-ratings of executive functioning for adults.

In this way, measures with valid psychometric properties are essential to assess organization abilities, to determine if intervention should be implemented, or to probe effectiveness of interventions, no matter which intervention is administered. There are several organization-specific measures so far (eg, Children's Organization Skills Scale [COSS] and On Time Management Organization and Planning [ON-TOP]). ${ }^{7,8}$ However, to our knowledge, there is no measure that can assess organizational abilities in Japan. Additionally, there is no measure that can probe organizational abilities in both childhood and adulthood. A measure that can be compatibly used for both childhood and adult would be clinically useful, especially for follow-up studies and assessment of adolescents, which is in the transition period from childhood to adulthood.

Japanese people have long been interested in methods for tidying things up due to their cultural background and small residential situation (Furoshiki and Futon are good examples for this). Recently, many books on decluttering or organizing have been published in Japan. In keeping with this trend, Marie Kondo's The life changing magic of tidying up became a bestseller in Europe and the US as well as in Japan, and she was selected as one of the 100 most influential people in Time. ${ }^{9}$ This fact revealed that there is worldwide growing interest in organization among people. It would be useful if there is a scale that can judge severity of disorganization not only for individuals with ADHD but also for those without ADHD.

In this study, the reliability and validity of the originally developed self-rating organization scale (SOS) were tested in a group of adults with ADHD and a group of control university students and adults. Furthermore, cutoff scores of factors in SOS were set to determine the clinical level of disorganization.

\section{Patients and methods Developing the SOS}

The SOS was developed largely referring to the child organization scale (COS). The COS was developed to assess children's organizational abilities and disorganization of youth with ADHD. ${ }^{10}$ Among several scales related to organization, the COS was chosen as a prototype of the SOS since the questions in the COS are relatively small in number and could be easily transformed into compatible ones with both children and adults. The 26 questions in the COS were divided into two subdomains: organization of objects and organization of time. The former contained 15 items related to various aspects of organizational placement or inanimate objects. The latter consisted of eleven items related to temporal organization. To prevent response bias, ten items were reverse scored. There were three dummy items that probe how he/she attributes his/her disorganization to others (eg, family and classmates) in the COS. All questions were rated on a five-point Likert scale of frequency.

The first author contacted one of the developers of the COS to obtain permission to use it and to confirm if there is substantial difference in meaning when items were translated into Japanese. On top of that, several questions were originally added to the COS. Item pools were generated from the literature, from professionals in the field, and from the clinical experience and compared to the contents of COS. As a result, three questions were adopted because it was frequent complaints of youths and adults with ADHD and uniquely reflecting a small residential environment in Japan. Furthermore, questions in the COS were transformed so that adults 
as well as youth can answer them (eg, "I have trouble finding my things in school when I need them." was transformed into "I have trouble finding my things in school or workplace when I need them."). The final tentative question list of the SOS has 29 questions, including three dummy questions.

\section{Participants}

The present study had a total of 1,017 participants divided into three groups: 1) ADHD group (47 adults with ADHD, 25 males, mean age $=30.70 \pm 8.51$ years, age range $=19-50$ years $)$, 2) adult (control) group (113 adults, 45 males, mean age $=34.18 \pm 10.69$, age range $=20-65$ years $)$, and 3 ) student (control) group (857 university students, 463 males, mean age $=20.12 \pm 1.39$ years, age range $=18-45$ years $)$. Another 27 (2.5\%) participants (one, five, and 21 from the ADHD, adult, and student groups, respectively) who failed to answer at least one question in the SOS were not included in the three groups. The student group was made up from students in two universities, and the adult group was recruited from several Japanese companies or facilities.

The ADHD group was composed of outpatients at the clinics where the first author was working. A clinical diagnosis of ADHD was made by the Japanese semi-structured diagnostic interview for adult ADHD. According to the $D S M-V$ criteria, the ADHD group comprised 33 subjects with predominantly inattentive presentation and 14 subjects with combined presentation. All the participants in the ADHD group had an intelligence quotient (IQ) measured on the Japanese version of the Wechsler Adult Intelligence Scale-III (mean full-scale IQ $=98.21 \pm 12.46$ ).

All participants in the ADHD and non-ADHD clinical groups were administered the Japanese version of the MiniInternational Neuropsychiatric Interview (MINI) to evaluate psychiatric disorders (the Japanese version of the MINI for $D S M-V$ was not published at this point). Psychiatric disorders other than those included in the MINI were diagnosed in accordance with the $D S M-I V .^{11}$

The ADHD group had the following psychiatric disorders other than ADHD (one person can have more than one disorder): major depressive disorder (12), dysthymic disorder (12), social anxiety disorder (two), autism spectrum disorder (ASD, three), obsessive-compulsive disorder (two), posttraumatic stress disorder (two), developmental coordination disorder (one), tic disorder (one), generalized anxiety disorder (one), and substance use disorder (alcohol, one).

All the subjects gave written informed consent, and patient anonymity has been preserved. This study was approved by the ethical committee of the Ryukoku University.
The Assessment System for individuals with ADHD (ASIA)

At the clinics, the first author administered the ASIA, a Japanese semistructured diagnostic interview for adult ADHD. ${ }^{12}$ The ASIA ADHD criterion A, which corresponds to the $D S M-V$ ADHD criterion A, comprises 144 original questions that probe nine inattention symptoms and nine hyperactivity-impulsivity symptoms. The ASIA ADHD criteria $\mathrm{B}$ to $\mathrm{E}$ correspond to the $D S M-V$ ADHD criteria $\mathrm{B}$ to E. The ASIA ADHD criteria showed acceptable reliability and validity in 36 adults with ADHD and 24 adults without ADHD.

In this study, besides a diagnostic tool, two questions from the ASIA were used to test the concurrent validity of the SOS. Both the questions are items from questions asking about in which inattention symptom e in the DSM-V (difficulty organizing) within 6 months. The former asks possible patients "How often have you been pointed out by your supervisors, colleagues, cohabitants, and friends that you cannot manage your time or you cannot make your appointments or deadline?" The latter is "How often have you had difficulty in tidying up your room or desk or organizing things?"

\section{Revised Sheehan Disability Scale (SDS) for disorganization}

In the original SDS, participants rate their impairment in three areas: school/work, social life, and communication or function with the family on a scale of $0-10$, with 10 being the worst. ${ }^{13}$ Ratings 4-7 and 7-9 are categorized as having moderate and marked impairment, respectively. The psychometric properties of the SDS were proven to be satisfactory in various populations including ADHD. ${ }^{14}$ The authors revised it to assess how severe participants' materials or temporal organization problem generally affects their daily life. In this study, a rating 4 or over is judged as having clinical level impairment caused by a relevant problem (ie, materials and/or temporal organization-related impairment).

\section{Data analyses}

Data analyses were conducted using IBM SPSS Statistics 22 and AMOS 22 for Windows, with the threshold for statistical significance set at $P<0.05$ (two-tailed test).

Factor analysis and structural equation modeling (SEM) were used to define the items for the SOS. Correlation analyses (Pearson's correlation coefficient) and paired $t$-test were conducted for test-retest reliability. ANOVA and correlation analyses (Pearson's correlation coefficient) were conducted 
for validity. An receiver operating characteristic (ROC) curve was drawn to settle the cutoff point.

\section{Results}

\section{Factor analyses}

In the preliminary analysis of distribution of each item, several items had floor effect, but no items had ceiling effect (Table 1). An exploratory factor analysis was conducted on 26 items of the SOS, excluding three dummy items. The scree plot showed that three of those factors explain most of the variabilities because the line starts to straighten after four. Among three factors, factor 1 mostly consists of items that are related to materials organization, factor 2 mostly consists of items that are related to organization of time, and factor 3 consists of items that are also related to organization of objects, but messed up situations should be more appropriate expression.

Item 8 "I have difficulty getting to classes or meetings on time." was deleted because a content of this item is evidently related to organization of time but classified into factor 1 .
Conversely, item 10 "I put my homework or task in the same place in my notebook, textbook, or document." was deleted because the contents of this item are clearly related to organization of objects but classified into factor 2. Item 20 "I start projects or tasks but have a hard time finishing them." and item 22 "I often act or say things before I think." were originally in organization of time but classified into factor 1 by a factor analysis. However, because the contents of these items are somewhat related to organization of objects as well as organization of time, confirmatory factor analyses were performed to compare the goodness of fit between the scale that consists of all items without three dummy items and items 8 and 10 (Model I) and the scale that consists of all items without three dummy items and items 8, 10, 20, and 22 (Model II). As a result, Model I (comparative fit index $[\mathrm{CFI}]=0.91$, root mean square error of approximation $[\mathrm{RMSEA}]=0.06$, Akaike information criteria $[\mathrm{AIC}]=945.80$, and Browne-Cudeck criterion $[\mathrm{BCC}]=951.19$ ) has no less fit than Model II (CFI $=0.91$, RMSEA $=0.06$, $\mathrm{AIC}=1075.60$, and $\mathrm{BCC}=1082.11$ ) does. Thus, Model I was adopted and

Table I Comparison of tentative SOS items between three groups

\begin{tabular}{|c|c|c|c|c|c|c|c|c|c|}
\hline \multirow[t]{2}{*}{ Item } & \multicolumn{2}{|c|}{ ADHD $(n=47)$} & \multicolumn{2}{|c|}{ Adult $(n=I \mid 3)$} & \multicolumn{2}{|c|}{ Student $(n=857)$} & \multirow[t]{2}{*}{$\boldsymbol{F}$} & \multirow{2}{*}{$\begin{array}{l}\text { ADHD vs } \\
\text { adult }\end{array}$} & \multirow{2}{*}{$\begin{array}{l}\text { ADHD vs } \\
\text { student }\end{array}$} \\
\hline & $M$ & SD & $M$ & SD & $M$ & SD & & & \\
\hline I & 2.34 & 1.07 & 1.27 & 0.89 & 1.37 & 1.03 & 21.48 & $*$ & $*$ \\
\hline 2 & 2.68 & 1.12 & I.3। & 0.85 & 1.59 & 0.98 & 33.85 & $*$ & $*$ \\
\hline 3 & 2.45 & 1.35 & 1.66 & 1.28 & 1.58 & 1.08 & 13.67 & $*$ & $*$ \\
\hline 4 & 1.15 & 1.18 & 0.72 & 1.19 & I.0I & 1.09 & 4.04 & $*$ & \\
\hline 5 & 2.09 & I.27 & 1.42 & I.07 & 1.68 & 1.19 & 5.41 & $*$ & \\
\hline 6 & 2.15 & 1.23 & 1.36 & 1.30 & 1.67 & 1.27 & 6.57 & $*$ & $*$ \\
\hline 7 & 1.91 & 1.12 & 0.79 & 0.74 & 0.95 & 0.97 & 25.26 & $*$ & $*$ \\
\hline 8 & 1.15 & 1.00 & 0.99 & 1.09 & 1.08 & 1.00 & 0.53 & $*$ & $*$ \\
\hline 9 & 2.57 & 1.16 & 1.15 & 1.05 & 1.59 & 1.18 & 24.91 & $*$ & $*$ \\
\hline 10 & 2.40 & I. 14 & 1.19 & I.15 & 1.49 & 1.29 & 15.33 & $*$ & \\
\hline II & 2.26 & I.37 & 1.50 & 1.29 & 2.17 & 1.29 & 13.92 & $*$ & $*$ \\
\hline 12 & 2.13 & 1.24 & 1.79 & 1.12 & 1.62 & I. 15 & 4.94 & $*$ & $*$ \\
\hline 13 & 2.60 & 1.36 & 1.72 & 1.25 & 2.21 & 1.29 & 9.88 & $*$ & \\
\hline $14^{d}$ & I.55 & 1.28 & 0.87 & 1.04 & 0.80 & 0.94 & 13.64 & $*$ & $*$ \\
\hline 15 & 2.11 & $\mathrm{I} .48$ & 1.84 & 1.28 & 2.54 & 1.28 & 16.60 & $*$ & \\
\hline 16 & 2.70 & 1.33 & 2.30 & 1.01 & 2.64 & I. 14 & 4.68 & $*$ & \\
\hline 17 & 2.23 & 1.31 & 1.24 & 1.02 & 1.20 & 1.04 & 21.27 & $*$ & \\
\hline 18 & 2.28 & I.14 & 1.58 & 1.26 & 1.90 & 1.15 & 6.69 & $*$ & $*$ \\
\hline $19^{d}$ & 0.53 & 0.83 & 0.48 & 0.72 & 0.62 & 0.83 & 1.58 & & \\
\hline 20 & 2.51 & 1.18 & I.II & 0.86 & 1.32 & 0.98 & 37.04 & $*$ & $*$ \\
\hline 21 & 2.17 & 1.24 & 1.13 & 0.98 & I.34 & I.II & 15.30 & $*$ & $*$ \\
\hline 22 & 2.51 & 1.12 & 1.47 & 1.00 & 1.60 & 1.08 & 17.38 & $*$ & $*$ \\
\hline 23 & 2.26 & I.I5 & 1.26 & 0.75 & 1.67 & 0.98 & 18.77 & $*$ & $*$ \\
\hline 24 & 1.87 & 1.38 & 1.10 & 0.94 & 1.43 & I. 18 & 7.92 & $*$ & $*$ \\
\hline 25 & 2.32 & 1.30 & 1.33 & 1.26 & 1.45 & 1.22 & 12.01 & $*$ & $*$ \\
\hline $26^{d}$ & 0.60 & 1.12 & 0.30 & 0.55 & 0.53 & 0.75 & 4.95 & $*$ & $*$ \\
\hline 28 & 2.21 & 1.43 & I.II & 1.02 & 1.32 & 1.23 & 14.30 & $*$ & $*$ \\
\hline 29 & 2.13 & 1.42 & 1.78 & 1.05 & I.7I & 1.29 & 2.44 & & \\
\hline
\end{tabular}

Notes: *The ADHD group is significantly higher than the relevant group. $d$ indicates a dummy item.

Abbreviations: SOS, self-rating organization scale; ADHD, attention-deficit hyperactivity disorder; $M$, mean; SD, standard deviation. 
Table 2 Summary of exploratory factor analysis results for the SOS using maximum likelihood estimation $(n=1,017)$

\begin{tabular}{|c|c|c|c|}
\hline \multirow[t]{2}{*}{ Item } & \multicolumn{3}{|c|}{ Factor loadings } \\
\hline & I (MD) & II (TD) & III (Mess) \\
\hline 17 & 0.789 & 0.034 & -0.116 \\
\hline 7 & 0.700 & 0.051 & -0.064 \\
\hline 21 & 0.674 & 0.029 & 0.061 \\
\hline 3 & 0.668 & -0.030 & 0.027 \\
\hline 2 & 0.646 & 0.047 & 0.114 \\
\hline I & 0.621 & -0.009 & 0.189 \\
\hline 23 & 0.601 & -0.117 & 0.162 \\
\hline 27 & 0.512 & -0.001 & 0.126 \\
\hline 9 & 0.050 & 0.660 & -0.007 \\
\hline 5 & -0.171 & 0.654 & -0.055 \\
\hline 4 & 0.246 & 0.631 & -0.284 \\
\hline 6 & 0.144 & 0.602 & 0.053 \\
\hline 18 & 0.189 & 0.598 & 0.103 \\
\hline 16 & -0.302 & 0.592 & 0.117 \\
\hline 12 & 0.206 & 0.532 & -0.211 \\
\hline 15 & -0.235 & 0.440 & 0.131 \\
\hline 13 & -0.014 & -0.036 & 0.799 \\
\hline 28 & 0.068 & 0.049 & 0.731 \\
\hline 24 & 0.094 & 0.023 & 0.728 \\
\hline 29 & 0.101 & -0.164 & 0.621 \\
\hline 25 & 0.245 & -0.024 & 0.567 \\
\hline II & -0.172 & 0.454 & 0.492 \\
\hline Eigen values & 5.76 & 2.89 & 1.57 \\
\hline$\%$ of variance & 26.18 & 13.14 & 7.15 \\
\hline Factor correlations & 1 & II & III \\
\hline 1 & - & 0.110 & 0.450 \\
\hline ॥ & & - & 0.230 \\
\hline III & & & - \\
\hline
\end{tabular}

Note: Factor loadings $>0.40$ appear in bold.

Abbreviations: SOS, self-rating organization scale; MD, materials disorganization; TD, temporal disorganization.

three factors were labeled as MD, Mess, and TD. Table 2 shows the factor loading of each item in the final version of the SOS (Table S1).

\section{Reliability}

The internal consistency reliability $(\alpha)$ of the SOS total (all items without three dummy items), MD, Mess, TD, and dummy scores were $0.82,0.86,0.80,0.73$, and 0.63 , respectively, across all the 1,017 participants.

As for test-retest reliability, in the 83 (ADHD $=18$, adult $=15$, and student $=50$ ) participants, the SOS total score at the first time point was significantly correlated with total score at the second time point $(r=0.82, P=0.000)$ and did not differ significantly between the first (mean $=33.99 \pm 11.50$ ) and second (mean $=33.70 \pm 10.75)$ ratings $(d f=82$, paired $t=0.39, P=0.70)$.

\section{Validity}

As shown in Tables 1 and 3, the ADHD group is significantly higher than the other two groups in terms of total score, all the factor scores, and the 17 out of 25 individual items.

The question related to organization of objects in the ASIA showed moderate correlation with factors MD $(n=76$ [ADHD $=47$, adult $=19$, and student $=12], r=0.65, P=0.000$ ) and Mess $(n=76, r=0.72, P=0.000)$, whereas the question related to organization of time in ASIA showed mild correlation with factor TD $(n=76, r=0.37, P=0.001)$.

The revised SDS score, which probes impairment in organization of objects, showed moderate correlation with factors $\mathrm{MD}(n=28[\mathrm{ADHD}=19$, adult $=9], r=0.52, P=0.006)$ and Mess ( $n=28, r=0.65, P=0.000)$, whereas the revised SDS score, which probes impairment in organization of time, showed mild correlation with factor TD $(n=28$, $r=0.31, P=0.12$ ).

\section{Cutoffs}

First, judging from the revised SDS score (score 4 or over), 15 and 10 (out of 28) were classified as having clinical level impairment in organization of objects and time, respectively. Mainly referencing ROC curve made from these groups (impairment and nonclinical/normal) and the scores of SOS. And the scores from ROC curve indicate the cutoff point that expects the impairment group more properly. Second, complementally consulting the mean score of the SOS in the ADHD group and the mean plus $1 \mathrm{SD}$ score of the

Table 3 Comparison of total and factor scores of the SOS between three groups

\begin{tabular}{|c|c|c|c|c|c|c|c|c|c|}
\hline & \multicolumn{2}{|c|}{ ADHD $(n=47)$} & \multicolumn{2}{|c|}{ Adult $(n=|| 3)$} & \multicolumn{2}{|c|}{ Student $(n=857)$} & \multirow[t]{2}{*}{$F$} & \multirow{2}{*}{$\begin{array}{l}\text { ADHD vs } \\
\text { adult }\end{array}$} & \multirow{2}{*}{$\begin{array}{l}\text { ADHD vs } \\
\text { student }\end{array}$} \\
\hline & $M$ & SD & $M$ & SD & $M$ & SD & & & \\
\hline Total & 48.59 & 14.12 & 30.15 & 12.17 & 35.44 & 11.98 & 37.78 & $*$ & $*$ \\
\hline MD & 18.04 & 7.30 & 9.54 & 5.26 & 10.48 & 5.62 & 42.46 & $*$ & $*$ \\
\hline Mess & 13.38 & 5.18 & 8.44 & 4.83 & 10.28 & 5.27 & 15.29 & $*$ & $*$ \\
\hline TD & 17.17 & 4.83 & 12.17 & 5.55 & 14.68 & 5.55 & $|8.4|$ & $*$ & $*$ \\
\hline Dummy & 2.68 & 2.49 & 1.65 & 1.81 & 1.94 & 1.92 & 4.90 & $*$ & $*$ \\
\hline
\end{tabular}

Note: *The ADHD group is significantly higher than the relevant group.

Abbreviations: SOS, self-rating organization scale; ADHD, attention-deficit hyperactivity disorder; MD, materials disorganization; TD, temporal disorganization. 
SOS in the other two groups (Table 1), the cutoffs for three factors were set in view of the necessary balance between sensitivity and specificity. Finally, the best cutoffs for differentiating between clinical level impairment and nonclinical/ normal level impairment on the MD, Mess, and TD were 16 (area under the curve $[\mathrm{AUC}]=0.76$, sensitivity $=0.73$, and specificity $=0.67), 16$ (AUC $=0.81$, sensitivity $=0.60$, and specificity $=0.75)$, and 18 (AUC $=0.70$, sensitivity $=0.70$, and specificity $=0.65)$, respectively.

\section{Discussion}

In this study, the SOS compatible to both youth and adult was developed referring to the existing scale and our clinical experience. Four items were deleted from the 29 tentative items based on the results of exploratory and confirmatory factor analyses. As a result, three factors, MD, TD, and Mess, were extracted. The internal consistency of the final version of the SOS was $\sim 0.80$, the correlation coefficient of two ratings with 2 -week interval was $>0.80$, and there was no significant difference between two ratings. The total score, three factor score, and most of the item scores in the ADHD group were significantly higher than those in the control groups. Factor scores were mild-to-moderately correlated with related question scores in the semistructured diagnostic interview for adult ADHD. Cutoffs were set using a revised disability scale for organization of objects and time. Their sensitivity and specificity range from 0.60 and 0.65 to 0.73 and 0.75 , respectively.

Floor effects were found in some of the items; however, these items were not deleted from the subsequent analyses because part of the clinical utility of the scale remains, optimizing the potential for the clinician to identify rare but potentially clinically salient sources of impairment.

Dummy items, which were originally classified into organization of objects, were excluded from factor analysis but included in the final version of the SOS. There are three reasons for this: 1) the number of dummy items is only three, which would be too small to make an independent factor. 2) Although they were classified in organization of objects in the original scale, they were more related to attribution of disorganization to others than organization of objects itself. 3) The attribution of disorganization to others and its change is of great clinical importance. ${ }^{15}$ The SOS, which is made to be used for youth as well as adults, has strength in following the trajectory of attribution.

By factor analysis, three factors were extracted. The original scale was hypothesized to have two subdomains: organization of objects and time. Caution is needed that subdomains in the original version were made theoretically, not by factor analysis. Items in organization of objects in the original version are largely classified into two categories in this study, possibly because newly added three items, which reflect Japanese small residential situation, may probe a messed-up situation at home and this would give rise to a new factor (Mess). The COSS has three subscales, task planning, organized actions, and memory and materials management, and inconsistency index. ${ }^{7}$ In this respect, the Mess factor is very unique in the SOS. The environmental and cultural context affects the expression of functional impairments arising from ADHD symptoms. ${ }^{16}$ Japan has a unique cultural background that is different especially to western countries. The Mess factor would shed light on this unique aspect of Japanese culture.

Items 20 and 22, which were originally classified as organization of time, were also classified into MD. Items 20 and 22 would be more accurately labeled as executive function and impulsivity, respectively. Items 6 and 18 were originally classified into organization of objects; however, in this study, they were loaded into TD. A possible explanation is that both the questions imply a "Do Something Right Away", which is related to behaviors during the flow of time. Thus, it was decided to leave these items as TD in the SOS, but this decision could affect relatively low correlation between TD factor score and relevant ASIA question and revised SDS.

The Cronbach's $\alpha$ s of the SOS and its factors were all satisfactory. The SOS also showed satisfactory test-retest reliability, as indicated by significant $r$ value of 0.82 with no significant difference in scores between the first and second ratings.

The total score, three factor scores, and most of the item scores on the SOS were significantly higher in the ADHD group than the other two control groups, demonstrating discriminant validity.

The factor scores of the SOS were acceptably and significantly correlated with the score of relevant questions in the ASIA, which are usually more reliable than the score of self-rated questions. Thus, these results show that the SOS has concurrent validity.

Employing optimal cutoffs, the sensitivity, specificity, and positive and negative predictive value of the SOS were all $\geq 0.60$ and seem acceptable.

There are several limitations in this study. First of all, a one-size-fits-all approach has inevitably some shortcomings. It is well known that there is a difference between adult and child in symptoms and impairments. ${ }^{17}$ The COS on which the SOS was referred is an old scale developed $>20$ years ago. 
Although originally the ratio of reverse item is high in TD, the fact that all the items in TD are reverse items should be beyond coincidence. Being reverse items could affect the results of exploratory factor analysis somehow.

Because this scale was intended for use in clinical setting, the usefulness in monitoring the effect of intervention should be tested. Furthermore, other clinical correlates of disorganization should be investigated. Specifically, a unique factor Mess might be related to hoarding disorder newly introduced in the $D S M-V$ or depression. Finally, but most importantly, this scale should be validated with young samples.

In this study, the SOS was developed and validated with adult samples. There are three factors in this scale: MD, Mess, and TD. The reliability and validity in the SOS are acceptable. Additionally, cutoffs for three factors were set for clinical use. However, further study is necessary to enhance the clinical utility of the SOS.

\section{Acknowledgment}

This study was supported in part by the Grants-in-Aid for Scientific Research (C) (No 70596460) from the Japan Society for the Promotion of Science.

\section{Disclosure}

The authors report no conflicts of interest in this work.

\section{References}

1. Landberg JM, Epstein JN, Graham AJ. Organizational-skills interventions in the treatment of ADHD. Expert Rev Neurother. 2008;8(10): 1549-1561.

2. American Psychiatric Association. Diagnostic and Statistical Manual of Mental Disorders. 5th ed (DSM-5). Arlington, VA; Washington, DC: American Psychiatric Association; 2013.

3. Willcutt EG. The prevalence of DSM-IV attention-deficit/hyperactivity disorder: a meta-analytic review. Neurotherapeutics. 2012;9(3): 490-499.
4. Barkley RA. Behavioral inhibition, sustained attention, and executive functions: constructing a unifying theory of ADHD. Psychol Bull. 1997; 121(1):65-94.

5. Stevenson CS, Whitmont S, Bornholt L, Livesey D, Stevenson RJ. A cognitive remediation programme for adults with attention deficit hyperactivity disorder. Aust NZ J Psychiatry. 2002;36(5):610-616.

6. Baldwin RL, Chelonis JJ, Flake RA, et al. Effect of methylphenidate on time perception in children with attention-deficit/hyperactivity disorder. Exp Clin Psychopharmacol. 2004;12(1):57-64.

7. Abikoff $\mathrm{H}$, Gallagher R, Wells KC, et al. Remediating organizational functioning in children with ADHD: immediate and long-term effects from a randomized controlled trial. J Consult Clin Psychol. 2013;81(1): 113-128.

8. Solanto MV, Marks DJ, Mitchell K, Wasserstein J, Kofman MD. Development of a new psychosocial treatment for adults with $\mathrm{AD} / \mathrm{HD}$ J Attent Disord. 2008;11(6):728-736.

9. Time Magazine. The 100 Most Influential People. New York: Time Inc; 2015.

10. Zentall SS, Harper GW, Stormont-Spurgin M. Children with hyperactivity and their organizational abilities. J Educ Res. 1993;87: 112-117.

11. Otsubo T, Tanaka K, Koda R, et al. Liability and validity of Japanese version of the Mini-International Neuropsychiatric Interview. Psychiatry Clin Neurosci. 2005;59(5):517-526.

12. Takeda T, Tsuji Y, Uwatoko T, Kurita H. Reliability and validity of ADHD diagnostic criteria in the Assessment System for Individuals with ADHD (ASIA), a Japanese semi-structured diagnostic interview. BMC Psychiatry. 2015;15:130.

13. Yoshida T, Otsubo T, Tsuchida H, Wada Y, Kamijima K, Fukui K. Reliability and validity of the Sheehan Disability Scale-Japanese version. Jpn J Psychopharmacol. 2004;7:1645-1653.

14. Coles T, Coon C, DeMuro C, McLeod L, Gnanasakthy A. Psychometric evaluation of the Sheehan Disability Scale in adult patients with attention-deficit/hyperactivity disorder. Neuropsychiatr Dis Treat. 2014; 10:887-895.

15. Hoza B, Pelham WE, Waschbusch DA, Kipp H, Owens JS. Academic task persistence of normally achieving ADHD and control boys: performance, self-evaluations, and attributions. J Consult Clin Psychol. 2001;69(2):271-283.

16. Cheung KK, Wong IC, Ip P, et al. Experiences of adolescents and young adults with ADHD in Hong Kong: treatment services and clinical management. BMC Psychiatry. 2015;15:95.

17. Spencer TJ, Biederman J, Mick E. Attention-deficit/hyperactivity disorder: diagnosis, lifespan, comorbidities, and neurobiology. J Pediatr Psychol. 2007;32(6):631-642. 


\section{Supplementary material}

Table SI Self-rating organization scale

\begin{tabular}{|c|c|}
\hline Item & Description \\
\hline 1 & I have trouble finding my things in school or workplace when I need them. \\
\hline 2 & I forgot to do my jobs (task, homework, assistance, and choirs) at home. \\
\hline 3 & When I cannot find something I need, I quickly get upset. \\
\hline 4 & I show up on time for school or workplace. ${ }^{r}$ \\
\hline 5 & I make plans for what I am going to do after school or job.r \\
\hline 6 & I put my belongings in the same place when I come home from school or workplace. \\
\hline 7 & I do my homework or task but cannot find it when it is due. \\
\hline 8 & I have difficulty getting to classes or meetings on time.\# \\
\hline 9 & I have a plan for deciding which homework or task assignment to do first. ${ }^{r}$ \\
\hline 10 & I put my homework or task in the same place in my notebook, textbook, or document."\# \\
\hline 11 & At the end of the day, I hang up my clothes or put them away immediately after I take them off. $r$ \\
\hline 12 & I am one of the first people to be at a meeting place with my friends. ${ }^{r}$ \\
\hline 13 & I would keep my room messy, if I were allowed. \\
\hline 14 & My family puts things where I cannot find them..$^{d}$ \\
\hline 15 & When I have several things to do in a day, I make a list or put notes around. ${ }^{r}$ \\
\hline 16 & I make plans for what I am going to do at recess or lunch.r \\
\hline 17 & I lose things at school or workplace. \\
\hline 18 & After I use something, I put it back where it belongs. ${ }^{r}$ \\
\hline 19 & Other kids or colleagues lose my things. ${ }^{d}$ \\
\hline 20 & I start projects or tasks but have a hard time finishing them." \\
\hline 21 & I have trouble remembering where I put things at home that I need everyday (like keys). \\
\hline 22 & I often act or say things before I think.\# \\
\hline 23 & I do not realize that I have forgotten something until I am already at school or workplace. \\
\hline 24 & My clothes are crumpled and messy. \\
\hline 25 & I keep my school or job stuff in my desk at school or workplace messy. \\
\hline 26 & Kids at school or colleagues at workplace mess up my stuff. ${ }^{d}$ \\
\hline 27 & When I tidy up, some of the same items appear. \\
\hline 28 & There is no space to walk on the floor at home because I put plenty of things directly on the floor. \\
\hline 29 & Things pile up in my room because I have trouble in throwing things away. \\
\hline
\end{tabular}

Notes: Low scores indicated a lower occurrence of the behavior. Items with " $r$ " are reverse coded; a high score is equivalent to "never". Items with a "\#” were deleted from the scale. Items with " $d$ " are dummy items.

\section{Publish your work in this journal}

Neuropsychiatric Disease and Treatment is an international, peerreviewed journal of clinical therapeutics and pharmacology focusing on concise rapid reporting of clinical or pre-clinical studies on a range of neuropsychiatric and neurological disorders. This journal is indexed on PubMed Central, the 'PsycINFO' database and CAS, and is the official journal of The International Neuropsychiatric Association (INA). The manuscript management system is completely online and includes a very quick and fair peer-review system, which is all easy to use. Visit http://www.dovepress.com/testimonials.php to read real quotes from published authors. 